\title{
ORIGINAL ARTICLE \\ Screening for neuropathic pain after spinal cord injury with the Spinal Cord Injury Pain Instrument (SCIPI): a preliminary validation study
}

\author{
TN Bryce ${ }^{1}$, JS Richards ${ }^{2}$, CH Bombardier ${ }^{3}$, MP Dijkers ${ }^{1}$, JR Fann ${ }^{4}$, L Brooks ${ }^{5}$, A Chiodo ${ }^{6}$, \\ DG Tate ${ }^{6}$ and M Forchheimer ${ }^{6}$
}

Study design: Cross-sectional.

Objective: To preliminarily evaluate the validity of an interview-based spinal cord injury (SCl) neuropathic pain screening instrument. Setting: Six university-based $\mathrm{SCl}$ centers in the United States.

Methods: Clinician diagnoses of neuropathic pain (NP) and non-neuropathic pain subtypes were collected independently of descriptions of the pain characteristics provided by the persons with SCI by using the Spinal Cord Injury Pain Instrument (SCIPI); SCIPI information and physician diagnoses for 82 pain sites of which they were most confident were subsequently compared.

Results: Four of the SCIPI items correlated significantly with the NP subtype as determined by the clinician. The best cutoff score for identifying NP was an endorsement of two or more of these four items. Using this cutoff, sensitivity of the SCIPI was 78\%, specificity was $73 \%$ and overall diagnostic accuracy was $76 \%$.

Conclusion: In this preliminary study, the SCIPI, which can be administered by a nonclinician, appears to have good sensitivity, specificity and diagnostic accuracy in a $\mathrm{SCl}$ population; it may have a role as a screening tool for NP after SCl. Further study is needed.

Spinal Cord (2014) 52, 407-412; doi:10.1038/sc.2014.21; published online 11 March 2014

Keywords: pain; spinal cord injury; neuropathic; screening; reliability; validity

\section{INTRODUCTION}

Pain is common after spinal cord injury (SCI), with about $80 \%$ of individuals with SCI experiencing chronic pain and one third experiencing pain severe enough to interfere with daily activities. ${ }^{1,2}$ There are two major categories of pain that affect persons with SCI: nociceptive pain and neuropathic pain (NP). Nociceptive pain is defined as pain arising from the activation of peripheral nerve sensory receptors (nociceptors) capable of transducing and encoding noxious stimuli, whereas NP is defined as pain caused by a lesion or disease of the somatosensory nervous system. ${ }^{3,4}$ Non-neuropathic pain (NNP) includes both nociceptive and non-neuropathic non-nociceptive pains, where non-neuropathic non-nociceptive pain refers to pain that occurs when there is no identifiable noxious stimulus nor any detectable damage to the nervous system to which the pain can be attributed. ${ }^{5}$ The International Association for the Study of Pain (IASP) SCI Pain Classification, ${ }^{6}$ a predecessor of the International SCI Pain Classification, ${ }^{5}$ was developed to organize the different major pain types, and their subtypes, into an easily understandable taxonomy with clear definitions.

Although the IASP and the International SCI Pain Classification provide definitions and a common terminology for describing (sub)types of pain, their usage requires clinical expertise to elicit and interpret the signs and symptoms from a person with pain, and to match them to the specific taxonomic categories. To simplify this process and allow those with limited expertise to screen persons for a specific major pain type, over the years a number of instruments have been developed that aim to differentiate NP from NNP. These screening tools generally are based either on a combination of physical exam findings and interview/self report questions, or on interview/self report alone. Validation typically consists of comparing the judgment of an experienced clinician as to the nature of a pain to the prediction of the pain type (generally: NP vs NNP) produced by the screening tool.

Validated screening tools include the Leeds Assessment of Neuropathic Symptoms and Signs (LANSS), the self-report LANSS , the Douleur Neuropathique 4 questions (DN4), the Neuropathic Pain Questionnaire (NPQ), ID Pain, the Standardized Evaluation of Pain and the painDETECT Questionnaire (PDQ). ${ }^{7-13}$ The DN4, LANSS and Standardized Evaluation of Pain rely on physical exam findings in addition to interview or self report; the others only require interview or self report. Evaluations of the various screening tools have been based on data from samples of persons with heterogeneous etiologies

\footnotetext{
${ }^{1}$ Department of Rehabilitation Medicine, Icahn School of Medicine at Mount Sinai, The Mount Sinai Medical Center, New York, NY, USA; ${ }^{2}$ Department of Physical Medicine and Rehabilitation, University of Alabama at Birmingham, Birmingham, AL, USA; ${ }^{3}$ Department of Rehabilitation Medicine, University of Washington, Seattle, WA, USA; ${ }^{4}$ Department of Psychiatry and Behavioral Sciences, University of Washington, Seattle, WA, USA; ${ }^{5}$ Department of Rehabilitation Medicine, University of Miami Leonard M. Miller School of Medicine, Miami, FL, USA and ${ }^{6}$ Department of Physical Medicine and Rehabilitation, University of Michigan, Ann Arbor, MI, USA

Correspondence: Dr TN Bryce, Department of Rehabilitation Medicine, Icahn School of Medicine at Mount Sinai, The Mount Sinai Medical Center, 5 East 98 th Street, Box 1240B, New York, NY 10029-6574, USA.

E-mail: thomas.bryce@mssm.edu
}

Received 11 September 2013; revised 10 January 2014; accepted 25 January 2014; published online 11 March 2014 
of NP. Owing to the fact that peripheral causes of NP are more prevalent than the central ones, peripheral NP is present in a high proportion of cases in all the published development and validation samples. In these samples, the sensitivity (ability of the instrument to correctly identify a pain as NP) ranges from $67 \%$ for the NPQ to $83 \%$ for the DN4 and to $85 \%$ for the LANSS and the PDQ. ${ }^{7,9-11}$ Specificity (ability of the instrument to correctly identify a pain as not being NP) ranges from $74 \%$ for the NPQ to $80 \%$ for the LANSS and the PDQ, and to $90 \%$ for the DN4; whereas diagnostic accuracy (the overall proportion of correct classifications of the pain) ranges from $71 \%$ for the NPQ to $82 \%$ for the LANSS and to $86 \%$ for the DN4. ${ }^{7,9-11}$

These measures have sometimes demonstrated weaker psychometric properties when applied to homogeneous samples such as people with SCI-related pain. When tested in a group with SCI in Sweden, the sensitivity of the various tools ranged from $36 \%$ for the LANSS to $93 \%$ for the DN4; ${ }^{14}$ the NPQ and PDQ had intermediate values. ${ }^{14}$ The specificity ranged from $75 \%$ for the DN4 to $100 \%$ for the LANSS; whereas diagnostic accuracy ranged from $55 \%$ for the LANSS to $88 \%$ for the DN4. ${ }^{14}$ A number of factors might explain the decreased accuracy of the various screening tools in a SCI sample. One is that persons with SCI often experience pain in insensate areas.

In light of the need for a screening tool that is both sensitive and specific in differentiating NP from NNP in the SCI population, and can be administered by interview/self report, the Spinal Cord Injury Pain Instrument (SCIPI) was developed. On the basis of clinical experience and analysis of the literature, ${ }^{5,6,15-19}$ the primary author compiled a list of seven characteristics associated with NP after SCI (see Appendix A for a description of the items). The first three items concerned the pain descriptors that are most commonly associated with NP after SCI. The fourth addressed the presence of pain evoked by dynamic touch. The fifth item concerned exacerbating and ameliorating factors for NP, whereas the sixth related to the temporal presence of pain. The final item identified pain that was felt in insensate areas. With regard to the items included in the SCIPI, there is a significant overlap with items seen in other screening tools. The DN4, LANSS, NPQ, PD-Q and the SCIPI all include either an interview question (NPQ, PD-Q, SCIPI) or a physical finding on sensory examination (DN4, LANSS) related to the skin being sensitive to touch ${ }^{7,9-11}$ and all include questions related to the descriptors: burning and/or cold, electric shock-like and pins/needles/tingling. ${ }^{7,9-11}$ The characteristics of the pain being constant, unchanged with movement and occurring in insensate areas are found only in the SCIPI, although the DN4 does include an item of decreased sensation to stimuli on sensory examination. ${ }^{9}$

The aim of this study was to preliminarily assess the psychometric properties of the SCIPI, obtaining an initial indication of its utility in differentiating NP from NNP in persons with SCI.

\section{MATERIALS AND METHODS}

\section{Subjects}

All subjects were participants in a multicenter randomized, placebo-controlled trial of the extended-release antidepressant drug venlafaxine hydrochloride for the treatment of depression after SCI. Because of the close association of depression and pain, some data on pain were collected but evaluation of SCIPI was not a primary aim of the depression-treatment study. However, the authors recognized and took advantage of the opportunity to gather preliminary psychometric data on the SCIPI, specifically its correlation with clinically derived assessment of SCI pain subtype. The inclusion criteria for the larger study were: SCI of any level, American Spinal Injury Association Impairment Scale A-D, age 18-65 years, at least one month post SCI, undergoing first inpatient rehabilitation or living in the community, Patient Health Questionnaire-9 depression screen score $\geqslant 10$ twice a week apart and Diagnostic and Statistical Manual of Mental Disorders IV criteria fulfilled for major depression or dysthymia. The key exclusion criteria included undergoing other treatments for depression, suicidal intent or plan, current alcohol or drug dependence, and not being on stable doses of psychoactive medications. For the study reported here, the additional inclusion criterion was the presence of pain of any type that had persisted over seven days before screening.

\section{Study design}

The larger study was carried out in six SCI centers in the United States. The pain assessment portion of the protocol was conducted at five of the sites and consisted of two steps. First, during the baseline assessment a research assistant administered the SCIPI to each subject and scored it (see Appendix A for items). The research assistant also asked the subject to rate pain severity on a Numeric Rating Scale, ranging from $0=$ no pain to $10=$ worst pain imaginable. Both of these were done separately for each pain the subject reported (characterized by anatomical location), up to a maximum of three, in accordance with the International Basic Pain Data Set. ${ }^{20}$ Training of the research assistants occurred via phone teleconferences that included instruction in SCIPI administration and scoring. Second, a form with the three worst pain sites identified by the subject, and presented in descending order of pain severity, was provided to an independent clinician classifier (CC). The CCs were asked only to determine, as part of a clinical examination, whether each pain site was unequivocally neuropathic or 'other', and to provide a rating 1-5 that reflected how confident they were in their diagnosis, with five reflecting high confidence. All clinicians were physicians who were experienced in the clinical diagnosis of SCI pain and in the use of the IASP classification system. They were provided a written synopsis of the IASP classification system on which they were directed to base their assessments. The CCs were blinded to the SCIPI data acquired by the research assistants.

\section{Validity determination}

CC determinations of pain subtype using the IASP scheme were used as the reference standard with which the SCIPI-based characterizations were compared. However, as the CCs were not always certain of their diagnostic conclusion, only pain-site diagnoses with certainty ratings of four or five (the two highest levels of certainty) were used in parts of the analysis. Sensitivity, specificity and overall diagnostic accuracy were derived from comparison of the SCIPI with IASP-defined NP with the NNP classifications.

\section{Statistical analysis}

A principal component analysis with varimax rotation was applied to pointbiserial correlations between the draft SCIPI items, separately for the pain sites where the physician was confident in his/her designation of the nature of the pain as NP vs NNP, and for all pain sites. These exploratory analyses were repeated for a subset of four items (a, b, c and g) that were found to be most strongly correlated with the IASP classifications. The other three original SCIPI items were eliminated because they did not correlate significantly with IASP ratings.

A receiver-operating characteristic (ROC) curve was constructed to help determine the optimal discrimination threshold of the four SCIPI items in terms of maximizing overall diagnostic accuracy.

The statistical software packages used for all analyses were SPSS 17.0 (SPSS Inc., Chicago, IL, USA) and SAS 9.3. (SAS Institute Inc., Cary, NC, USA).

\section{RESULTS}

\section{Subject demographics}

Thirty-six subjects reported a total of 102 different pain sites for which both SCIPI and IASP data were collected. However, only 82 of the pain sites were accompanied by CC confidence ratings of four or five, indicating a high degree of confidence in the diagnosis, and were included in the final sample. The five most commonly reported pain 
Table 1 Demographic and clinical characteristics of the final sample of subjects with pain(s) characterized with high confidence

\begin{tabular}{|c|c|}
\hline Characteristic & $\mathrm{N}$ (Percent) or Mean (s.d.) \\
\hline Number of subjects & 36 \\
\hline Number of pain sites & 82 \\
\hline Age (years) (mean \pm s.d.) & $41.9(12.6)$ \\
\hline Male & $26(72 \%)$ \\
\hline Time since injury (years) (mean \pm s.d.) & $10.9(11.1)$ \\
\hline Average number of pains & $2.3(0.7)$ \\
\hline Average pain intensity $(0-10)$ & $6.0(2.0)$ \\
\hline \multicolumn{2}{|l|}{ SCl level } \\
\hline Cervical & $15(42 \%)$ \\
\hline Thoracic & $18(50 \%)$ \\
\hline Lumbar & $3(8 \%)$ \\
\hline Sacral & $0(0 \%)$ \\
\hline \multicolumn{2}{|l|}{ SCl completeness_AIS } \\
\hline A (complete) & $21(58 \%)$ \\
\hline B-incomplete (sensory) & $4(11 \%)$ \\
\hline C-incomplete (grade 0-2) & $1(3 \%)$ \\
\hline D_incomplete (grade $3+$ ) & $10(28 \%)$ \\
\hline \multicolumn{2}{|l|}{ Cause of injury } \\
\hline Fall & $4(11 \%)$ \\
\hline Vehicular & $18(50 \%)$ \\
\hline Violence & $6(17 \%)$ \\
\hline Other & $8(22 \%)$ \\
\hline
\end{tabular}

sites were: low back (16\%), legs (14\%), shoulders (11\%), neck (11\%) and feet $(6 \%)$. Clinical and demographic details of the participants are listed in Table 1. The sample was $72 \%$ male, with an average age of 41.9 years; $42 \%$ had cervical injuries and $58 \%$ had complete injures.

\section{Pain characteristics}

Thirty-three pains were determined to be NP by the clinicians (Table 2). Average pain intensity was similar for pains judged to be NP vs NNP. Endorsement of the SCIPI items by participants was high only for item $\mathrm{f}$ ('constant'), which was checked for slightly over half of all pains. Items a ('electrical or shock-like') and g ('occurs at insensate area') were endorsed for only about a quarter of the pain sites.

\section{Principal components analysis}

Only one strong inter-item correlation was evident (Table 3), which was between items a ('electrical or shock-like') and b ('pins and needles' or 'tingling'). Principal component analysis for the 102 pains showed three components with an eigenvalue over 1.00, which in combination explained $59 \%$ of the variance, after varimax rotation (Table 4). The results for the analysis for the 82 pains were similar, but the factors were different. Analysis of just the subset of four items similarly did not result in strong factors, and the factors found were discrepant.

\section{Discriminant properties of the items}

Four of the seven original SCIPI items were very clearly correlated with IASP classifications by the CCs $(P<0.05)$, whereas three items did not correlate at all $(P>0.6)$ (Table 2$)$. The ROC curve indicated that the best indicator of NP was the endorsement of any two of the four items reflective of NP (Figure 1).
Table 2 Pain site characteristics by IASP classification of NP and NNP (confidence level 4 and 5 only)

\begin{tabular}{|c|c|c|c|c|}
\hline \multirow[t]{2}{*}{ Baseline SCIPI } & \multicolumn{4}{|c|}{ IASP classification } \\
\hline & $N N P$ & $N P$ & Total & Sig \\
\hline Pain sites $(N)$ & $49(60 \%)$ & $33(40 \%)$ & $82(100 \%)$ & - \\
\hline \multicolumn{5}{|l|}{ Confidence in diagnosis } \\
\hline Certainty 5 & $65 \%$ & $67 \%$ & $66 \%$ & 1.000 \\
\hline Certainty 4 & $35 \%$ & $33 \%$ & $34 \%$ & \\
\hline \multicolumn{5}{|c|}{ Endorsement of SCIPI characteristics (\% yes) } \\
\hline a. electrical/shock like? & $5(10 \%)$ & $13(39 \%)$ & $18(22 \%)$ & 0.003 \\
\hline b. pins/needles, tingling? & $14(29 \%)$ & $24(73 \%)$ & $38(46 \%)$ & 0.000 \\
\hline c. skin feels hot/burning, cold? & $10(20 \%)$ & $17(52 \%)$ & $27(33 \%)$ & 0.004 \\
\hline d. skin is touch sensitive? & $14(29 \%)$ & $13(39 \%)$ & $27(33 \%)$ & 0.344 \\
\hline e. unchanged with movement? & $20(41 \%)$ & $16(48 \%)$ & $36(44 \%)$ & 0.506 \\
\hline f. pain constant? & $28(57 \%)$ & $22(67 \%)$ & $50(61 \%)$ & 0.490 \\
\hline g. occurs in insensate areas? & $8(16 \%)$ & $14(42 \%)$ & $22(27 \%)$ & 0.012 \\
\hline \multicolumn{5}{|l|}{ Number of items endorsed $(0-7)$} \\
\hline Overall & $1.7 \pm 1.2$ & $4.1 \pm 1.3$ & $2.7 \pm 1.7$ & 0.000 \\
\hline Certainty 5 pains only & $1.5 \pm 1.3$ & $4.3 \pm 1.0$ & $2.8 \pm 1.5$ & 0.000 \\
\hline Certainty 4 pains only & $2.1 \pm 0.9$ & $3.7 \pm 1.6$ & $2.6 \pm 1.8$ & 0.002 \\
\hline \multicolumn{5}{|l|}{ Average pain intensity (0-10 NRS) } \\
\hline All pain sites & $5.9 \pm 2.0$ & $6.1 \pm 1.9$ & $6.0 \pm 2.0$ & 0.616 \\
\hline Pain site \#1 & $6.9 \pm 1.7$ & $6.6 \pm 1.6$ & $6.8 \pm 1.6$ & 0.613 \\
\hline Pain site \#2 & $5.7 \pm 2.0$ & $5.6 \pm 2.2$ & $5.7 \pm 2.0$ & 0.986 \\
\hline Pain site \#3 & $4.6 \pm 1.9$ & $5.9 \pm 1.9$ & $5.2 \pm 1.9$ & 0.172 \\
\hline
\end{tabular}

Abbreviation: Sig, statistical significance.

Table 3 Point biserial correlations between SCIPI items for all pain sites (below the diagonal, $N=102$ ), and for pain sites which the physician was confident classifying the pain as NP or NNP (above the diagonal, $N=82$ )

\begin{tabular}{lcccccccc}
\hline & & $a$ & $b$ & $c$ & $d$ & $e$ & $f$ & $g$ \\
\hline Electrical/shock-like & a & - & 0.57 & 0.01 & 0.19 & 0.01 & 0.06 & 0.14 \\
Pins/needles, tingling & $\mathrm{b}$ & 0.53 & - & 0.29 & 0.23 & -0.08 & 0.14 & 0.27 \\
Skin feels hot/cold & $\mathrm{c}$ & 0.15 & 0.30 & - & 0.23 & 0.06 & 0.24 & 0.22 \\
Skin is touch-sensitive & $\mathrm{d}$ & 0.04 & 0.20 & 0.26 & - & 0.01 & 0.08 & -0.07 \\
Unchanged with & $\mathrm{e}$ & 0.04 & -0.07 & 0.01 & 0.04 & - & 0.10 & 0.07 \\
movement & & & & & & & & \\
Constant & $\mathrm{f}$ & -0.01 & 0.10 & 0.12 & 0.05 & 0.02 & - & 0.20 \\
Occurs at insensate area & $\mathrm{g}$ & 0.21 & 0.22 & 0.20 & 0.01 & 0.08 & 0.12 & - \\
\hline
\end{tabular}

Validity

The sensitivity or the ability of the 4-item SCIPI to correctly identify a pain as NP as compared with the diagnosis of the CC using the IASP criteria was 0.72 (Table 5). The specificity or the ability of the SCIPI to correctly identify that a pain was not NP was 0.78 , whereas the overall proportion of correct classifications using the SCIPI was 0.76 .

\section{DISCUSSION}

The principal components analysis and item correlations indicate that the original seven SCIPI items are not strongly correlated with one 
another (except possibly items a and b), suggesting that there is not one single factor or a few underlying factors that explain responses. It seems that the pain experienced (here for NP and NNP pains, as for other pain syndromes, and for pain in general) is quite idiosyncratic, with different people experiencing discrepant symptoms, perhaps related to different underlying mechanisms, associated with what they have in common-pain intensity. This would suggest that if there is a value to the individual SCIPI items, they need to be used individually, and there is no benefit in combining them in subscales.

The comparison of NP with NNP pains showed that four SCIPI-specific pain descriptors are highly correlated with CC classifications of NP in persons with SCI. These include items concerning sensations of electric shock, tingling or pins and needles, hot/burning

Table 4 Summary of results of principal component analyses

\begin{tabular}{lcccc}
\hline Sample used & $\mathrm{N}=102$ & $\mathrm{~N}=82$ & $\mathrm{~N}=102$ & $\mathrm{~N}=82$ \\
\hline Items used & $\mathrm{a}-\mathrm{g}$ & $\mathrm{a}-\mathrm{g}$ & $\mathrm{a}, \mathrm{b}, \mathrm{c}, \mathrm{g}$ & $\mathrm{a}, \mathrm{b}, \mathrm{c}, \mathrm{g}$ \\
Range of communalities & $0.36-0.74$ & $0.27-0.78$ & $0.29-0.67$ & $0.49-0.86$ \\
Factors extracted & 3 & 3 & 1 & 2 \\
Pct of variance explained & 59 & 62 & 46 & 71 \\
Items with factor & & & & \\
loadings >0.4 & $\mathrm{a}, \mathrm{b}, \mathrm{g}$ & $\mathrm{a}, \mathrm{b}, \mathrm{g}$ & $\mathrm{a}, \mathrm{b}, \mathrm{c}, \mathrm{g}$ & $\mathrm{a}, \mathrm{b}$ \\
$\quad$ Factor 1 & $\mathrm{c}, \mathrm{d}$ & $\mathrm{c}, \mathrm{e}, \mathrm{f}, \mathrm{g}$ & - & $\mathrm{c}, \mathrm{g}$ \\
Factor 2 & $\mathrm{e}, \mathrm{f}, \mathrm{g}$ & $\mathrm{d}$ & - & - \\
Factor 3 & & &
\end{tabular}

or cold/freezing, and whether or not the pain occurs in an insensate area of the body. Other characteristics commonly thought to be associated with SCI-related NP, for example, constant pain, pain that doesn't change with movement or activity, and tactile allodynia, were not found to discriminate NP from NNP in this small sample.

The SCIPI, as developed and validated in the present study, can be interviewer administered or self administered. It consists of four questions; a score of 1 is given to each positive item and a score of 0 to each negative item. The total score is calculated as a sum of the four items with a score of 0 indicating NNP, a score of 1 indicating possible NP and a score of 2 or greater indicating probable NP. With regard to the use of the SCIPI in practice, if maximizing sensitivity is paramount, for example, as a first screening for a study, a cutoff of $\geqslant 1$ may be appropriate, whereas if specificity is more important a cutoff of $\geqslant 2$ may be more appropriate.

One reason for the discrepancies in sensitivity and diagnostic accuracy of the other screening tools-LANSS ${ }^{7}, \mathrm{PDQ}^{10}, \mathrm{DN} 4^{9}$, and

Table 5 Correspondence between NP pain diagnosis using SCIPI (with score $\geqslant 2$ cutoff) and expert diagnosis using IASP criteria (certainty 4 or 5 pains only)

\begin{tabular}{lccc} 
& NP present & NP absent & Total \\
\hline SCIPI positive for NP & 24 & 11 & 35 \\
SCIPI negative for NP & 9 & 38 & 47 \\
Total & 33 & 49 & 82 \\
\hline
\end{tabular}

Sensitivity: 0.72; specificity: 0.78 ; accuracy: 0.76 .

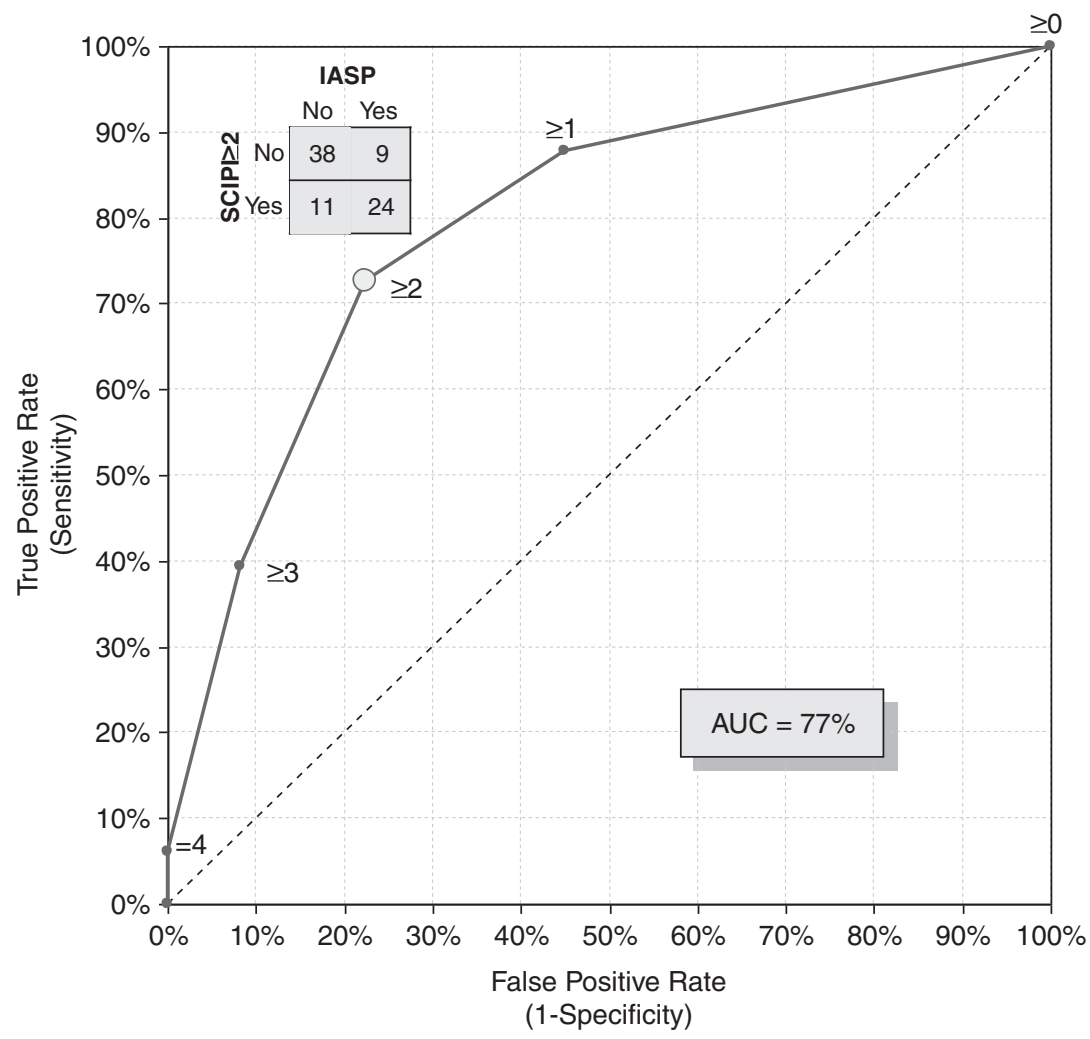

Figure 1 ROC curve for combination of SCIPI items. 
$\mathrm{NPQ}^{11}$ - between an SCI-only sample on the one hand and cohorts of varied neurological diagnoses on the other becomes apparent when one considers the individual items of these generic screening tools. For example, in the LANSS ${ }^{7}$ significant weight is given to the findings of evoked pain and skin changes common in peripheral causes of NP and complex regional pain syndrome, whereas persons with SCI are often insensate in areas where they have pain and rarely have skin changes associated with NP, rendering these questions moot for diagnosing SCI NP in cases where NP is almost certainly present.

The $\mathrm{DN} 4^{9}$ has two items on the clinical exam portion, hypoesthesia to touch and to pinprick at an area where the pain is located, which, although not originally intended to do so, provide the same information as that elicited by the last item of the SCIPI, that is, persons who experience pain in insensate areas below the level of injury by definition exhibit hypoalgesia to touch and pinprick in the same area. This finding may help explain the relative sensitivity of the DN4 as compared with the other measures in differentiating NP from NNP in persons with SCI in the Hallstrom study. ${ }^{14}$

Although it is true that the DN4 may have stronger overall psychometrics as compared with the SCIPI, it has to be administered in person. The benefit of the SCIPI is that it is possible to use it in situations where in-person screening is not available.

\section{Limitations}

Study limitations include the fact that all participants in this study were at least moderately depressed, which potentially limits the generalizability of the findings. Future studies of the psychometric qualities of the SCIPI should involve a broader cross-section of the SCI population. The diagnostic accuracy of the SCIPI as described here is based only on clinical classifications made with high confidence; had all the pain sites with less confidence on the part of the CCs as to diagnostic accuracy been used, the diagnostic accuracy of the SCIPI would likely have been lower. However, the reality of diagnosing NP and NNP pain in persons with SCI is that in many cases, clinical diagnostic judgments are not certain, for example, in individuals with incomplete injuries where both $\mathrm{NP}$ and NNP pain qualities are reported. Up to three pains per person were treated statistically as if they were independent observations, which is incorrect, of course, but considered not to be a major issue for this preliminary observation. Finally, there are no inter-rater reliability metrics on the expert classifications, no data on test-retest reliability of the SCIPI, and pain data were drawn from a small sample of subjects.

\section{CONCLUSIONS}

The results of the current study preliminarily suggest that answers to a simple yes/no questionnaire can be used to reliably distinguish NP from NNP in a group of depressed individuals with SCI and pain in which CCs are confident of their diagnosis. Nonclinicians can administer the SCIPI, which can also be self administered. The measure may be useful in the clinical research setting and in clinical practice as a simple means of screening for NP after SCI. More studies to further investigate the SCIPI's validity are recommended.

\section{DATA ARCHIVING}

There were no data to deposit.

\section{CONFLICT OF INTEREST}

The authors declare no conflict of interest.

\section{ACKNOWLEDGEMENTS}

The contents of this paper were developed under a grant from the National Institute on Disability and Rehabilitation Research, Department of Education (grant number H133A060107). However, these contents do not necessarily represent the policy of the Department of Education, and endorsement by the U.S. Federal Government should not be assumed.

1 Cardenas DD, Bryce TN, Shem K, Richards JS, Elhefni H. Gender and minority differences in the pain experience of people with spinal cord injury. Arch Phys Med Rehabil 2004; 85: 1774-1781.

2 Dijkers M, Bryce T, Zanca J. Prevalence of chronic pain after traumatic spinal cord injury: a systematic review. J Rehabil Res Dev 2009; 46: 13-29.

3 Loeser JD, Treede RD. The Kyoto protocol of IASP Basic Pain Terminology. Pain 2008; 137: 473-477.

4 Jensen TS, Baron R, Haanpaa M, Kalso E, Loeser JD, Rice AS et al. A new definition of neuropathic pain. Pain 2011; 152: 2204-2205.

5 Bryce TN, Biering-Sorensen F, Finnerup NB, Cardenas DD, Defrin R, Lundeberg T et al. International spinal cord injury pain classification: part I. Background and description. Spinal Cord 2012; 50: 413-417.

6 Siddall PJ, Yezierski RP, Loeser JD. Pain following spinal cord injury: clinical features, prevalence, and taxonomy. IASP Newsletter 2000; 3: 3-7.

7 Bennett M. The LANSS pain scale: the Leeds assessment of neuropathic symptoms and signs. Pain 2001; 92: 147-157.

8 Bennett MI, Smith BH, Torrance N, Potter J. The S-LANSS score for identifying pain of predominantly neuropathic origin: validation for use in clinical and postal research. J Pain 2005; 6: 149-158.

9 Bouhassira D, Attal N, Alchaar H, Boureau F, Brochet B, Bruxelle J et al. Comparison of pain syndromes associated with nervous or somatic lesions and development of a new neuropathic pain diagnostic questionnaire (DN4). Pain 2005; 114: 29-36.

10 Freynhagen R, Baron R, Gockel U, Tolle TR. PainDETECT: a new screening questionnaire to identify neuropathic components in patients with back pain. Curr Med Res Opin 2006; 22: 1911-1920.

11 Krause SJ, Backonja MM. Development of a neuropathic pain questionnaire. Clin J Pain 2003; 19: 306-314.

12 Portenoy R. Development and testing of a neuropathic pain screening questionnaire: ID Pain. Curr Med Res Opin 2006; 22: 1555-1565.

13 Scholz J, Mannion RJ, Hord DE, Griffin RS, Rawal B, Zheng $\mathrm{H}$ et al. A novel tool for the assessment of pain: validation in low back pain. PLOS Med 2009; 6: e1000047.

14 Hallstrom H, Norrbrink C. Screening tools for neuropathic pain: can they be of use in individuals with spinal cord injury? Pain 2011; 152: 772-779.

15 Siddall PJ, McClelland JM, Rutkowski SB, Cousins MJ. A longitudinal study of the prevalence and characteristics of pain in the first 5 years following spinal cord injury. Pain 2003; 103: 249-257.

16 Cardenas DD, Turner JA, Warms CA, Marshall HM. Classification of chronic pain associated with spinal cord injuries. Arch Phys Med Rehabil 2002; 83: 1708-1714.

17 Bryce TN, Ragnarsson KT. Epidemiology and classification of pain after spinal cord injury. Top Spinal Cord Inj Rehabil 2001; 7: 1-17.

18 Attal N, Fermanian C, Fermanian J, Lanteri-Minet M, Alchaar H, Bouhassira D. Neuropathic pain: are there distinct subtypes depending on the aetiology or anatomical lesion? Pain 2008; 138: 343-353.

19 Putzke JD, Richards JS, Hicken BL, Ness TJ, Kezar L, DeVivo M. Pain classification following spinal cord injury: the utility of verbal descriptors. Spinal Cord 2002; 40 : 118-127.

20 Widerstrom-Noga E, Biering-Sorensen F, Bryce T, Cardenas DD, Finnerup NB, Jensen MP et al. The international spinal cord injury pain basic data set. Spinal Cord 2008; 46: 818-823. 


\section{APPENDIX A}

Appendix A Initial SCIPI item pool

I am going to ask you a set of questions about each of the pain locations you mentioned. First I will ask you about the VERY WORST pain you have had in the last seven days. You said this pain was located answering the following questions. Please consider this pain when

\begin{tabular}{|c|c|c|}
\hline & \multicolumn{2}{|c|}{ Pain \# 1} \\
\hline & no & yes \\
\hline Is the quality of pain electrical or electric shock like? & 0 & 1 \\
\hline Is the quality of pain like pins and needles, or tingling? & 0 & 1 \\
\hline $\begin{array}{l}\text { Does the skin over the area of pain or inside your body where } \\
\text { the pain is located feel hot or burning or cold or freezing? }\end{array}$ & 0 & 1 \\
\hline
\end{tabular}

(Continued)

Pain \# 1

no yes

Is the skin over the area of pain abnormally sensitive to touch and without $\begin{array}{ll}0 & 1\end{array}$ any surgical scars, ulcers or breaks in the skin?

Is the pain usually unchanged with movement of the painful area?

$0 \quad 1$

$\begin{array}{lll}\text { Do you experience pain all the time without any breaks when you are } & 0 & 1\end{array}$

awake (although it may vary in intensity during different times)?

Does the pain only occur in an area of the body in which you have no $\quad \begin{array}{ll}0 & 1\end{array}$

feeling on the skin overlying that area? 